\title{
INVERSE EIGENVALUE PROBLEMS FOR PERTURBED SPHERICAL SCHRÖDINGER OPERATORS
}

\author{
ALEKSEY KOSTENKO, ALEXANDER SAKHNOVICH, AND GERALD TESCHL
}

\begin{abstract}
We investigate the eigenvalues of perturbed spherical Schrödinger operators under the assumption that the perturbation $q(x)$ satisfies $x q(x) \in$ $L^{1}(0,1)$. We show that the square roots of eigenvalues are given by the square roots of the unperturbed eigenvalues up to an decaying error depending on the behavior of $q(x)$ near $x=0$. Furthermore, we provide sets of spectral data which uniquely determine $q(x)$.
\end{abstract}

\section{INTRODUCTION}

Given a Schrödinger operator with a rotationally symmetric potential, separation of variables leads to the spherical Schrödinger operator (e.g., [15], [16])

$$
H=-\frac{d^{2}}{d x^{2}}+\frac{l(l+1)}{x^{2}}+q(x), \quad l=0,1,2, \ldots
$$

In this note we are interested in the case where the particle is confined to a finite ball (of radius 1 for notational simplicity — which can always be achieved by scaling $x$ ). This problem has attracted much interest in the past and several results concerning the eigenvalues of these problems have been derived. The first results are based on the seminal work by Guillot and Ralston [6] which deals with the case $l=1$ and $q \in L^{2}(0,1)$. It was later extended by Carlson [4, [5] and recently by Serier 14 who extended their results to arbitrary $l \in \mathbb{N}_{0}$. However, the assumption $q \in L^{2}(0,1)$ clearly excludes the physically interesting case of a Coulomb type singularity $q(x)=\frac{\gamma}{x}+\ldots$ This case was included for $l=0$ in the work of Savchuk and Shkalikov [12, [13 who considered $q \in W^{-1,2}(0,1)$. Their work was later extended by Albeverio, Hryniv, and Mykytyuk who first covered the case $q \in W^{-1, p}(0,1), p \in[1, \infty)$, for $l=0$ in [2] and later on extended this to all $l \in \mathbb{N}_{0}$ in [3] using the double commutation method. Their condition includes in particular the case $x q(x) \in L^{p}(0,1), p \in[1, \infty)$, which will be the condition we are interested in here.

Our main motivation for this paper is the paper by Zhornitskaya and Serov 18 who treat the general case $l \geq-\frac{1}{2}$ under the assumption $q \in L^{1}(0,1)$. We want to extend their results in several ways: First of all we will replace the condition $q(x) \in L^{1}(0,1)$ by $x q(x) \in L^{1}(0,1)$. Moreover, they show in [18] that the Dirichlet eigenvalues satisfy

$$
\mu_{n}=\left(j_{l+\frac{1}{2}, n}+\varepsilon_{n}\right)^{2}
$$

2000 Mathematics Subject Classification. Primary 34B20, 34L15; Secondary 81V45, 47A10. Key words and phrases. Schrödinger operators, spectral theory, strongly singular potentials. Inverse Problems 26, 105013, 14pp (2010).

Research supported by the Austrian Science Fund (FWF) under Grant No. Y330. 
where $j_{l+\frac{1}{2}, n}=\pi\left(n+\frac{l}{2}\right)+O\left(n^{-1}\right)$ are the zeros of the Bessel function $J_{l+1 / 2}(z)$ and the error satisfies $\left|\varepsilon_{n}\right| \leq \frac{\pi}{4}$ (this is claimed for all $n$ but only proven for large $n)$. We will show that the error satisfies

$$
\varepsilon_{n}=O\left(\int_{0}^{1} \frac{y|q(y)|}{1+n y} d y\right) .
$$

(For $l=-\frac{1}{2}$ one has to replace $q(y)$ by $(1-\log (y)) q(y)$.) In particular, for $q \in$ $L^{1}(0,1)$ we get $\varepsilon_{n}=O\left(n^{-1}\right)$ and for a Coulomb type singularity $q(x)=\frac{\gamma}{x}+L^{1}(0,1)$ we get $\varepsilon_{n}=O\left(n^{-1} \log (n)\right)$.

Based on this information we will give some sets of spectral data which, in addition to the Dirichlet spectrum, uniquely determine $q$ and $l$ again generalizing the corresponding result from [18] to the case $x q(x) \in L^{1}(0,1)$. Moreover, in their construction they use the fact that a certain Wronskian does not vanish. Unfortunately this Wronskian can indeed vanish (we will give a simple counter example due to Ralston in Remark 2.7) but we will show that the use of this fact can be avoided.

\section{THE SPHERICAL SCHRÖDINGER OPERATOR}

Our prototypical example will be the spherical Schrödinger operator given by

$$
H_{l}=-\frac{d^{2}}{d x^{2}}+\frac{l(l+1)}{x^{2}}, \quad x \in(0,1), \quad l \geq-\frac{1}{2} .
$$

Note that we explicitly allow non-integer values of $l$ such that we also cover the case of arbitrary space dimension $n \geq 2$, where $l(l+1)$ has to be replaced by $l(l+n-2)+(n-1)(n-3) / 4$ [16, Sec. 17.F].

With the usual boundary conditions at $x=0$ (for $\left.l \in\left[-\frac{1}{2}, \frac{1}{2}\right)\right)$ and $x=1$

$$
\lim _{x \rightarrow 0} x^{l}\left((l+1) f(x)-x f^{\prime}(x)\right)=0, \quad f(1)=0 \text { or } f^{\prime}(1)+\beta f(1)=0,
$$

it gives rise to a self-adjoint operator in the Hilbert space $L^{2}(0,1)$. Two linearly independent solutions of

$$
-f^{\prime \prime}(x)+\frac{l(l+1)}{x^{2}} f(x)=z f(x)
$$

are given by (see [1, (9.1.49)])

$$
\theta_{l}(z, x)=-z^{\frac{2 l+1}{4}} \sqrt{\frac{\pi x}{2}} \begin{cases}\frac{-1}{\sin \left(\left(l+\frac{1}{2}\right) \pi\right)} J_{-l-\frac{1}{2}}(\sqrt{z} x), & l+\frac{1}{2} \in \mathbb{R}_{+} \backslash \mathbb{N}_{0}, \\ Y_{l+\frac{1}{2}}(\sqrt{z} x)-\frac{1}{\pi} \log (z) J_{l+\frac{1}{2}}(\sqrt{z} x), & l+\frac{1}{2} \in \mathbb{N}_{0},\end{cases}
$$

where $J_{l+1 / 2}$ and $Y_{l+1 / 2}$ are the usual Bessel and Neumann functions. All branch cuts are chosen along the negative real axis unless explicitly stated otherwise. If $l$ is an integer they of course reduce to spherical Bessel and Neumann functions and can be expressed in terms of trigonometric functions (cf. e.g. 1, 17] and also [15, Sect. 10.4]). 
Using the power series for the Bessel and Neumann functions one verifies that they have the form

$$
\theta_{l}(z, x)=\frac{\Gamma\left(l+\frac{3}{2}\right) 2^{l+1}}{x^{l} \sqrt{\pi}} \begin{cases}\frac{1}{2 l+1} g_{l}\left(z x^{2}\right), & l+\frac{1}{2} \in \mathbb{R}_{+} \backslash \mathbb{N}_{0}, \\ \frac{1}{2 l+1} g_{l}\left(z x^{2}\right)-\frac{\left(z x^{2}\right)^{l+\frac{1}{2}} \log (x)}{\Gamma\left(l+\frac{3}{2}\right)^{2} 2^{2 l+1}} f_{l}\left(z x^{2}\right), & l+\frac{1}{2} \in \mathbb{N}, \\ (\log (2)-\gamma) g_{l}\left(z x^{2}\right)-\log (x) f_{l}\left(z x^{2}\right), & l=-\frac{1}{2},\end{cases}
$$

where $f_{l}(z), g_{l}(z)$ are entire functions with $f_{l}(0)=g_{l}(0)=1$ and $\gamma$ is the EulerMascheroni constant.

In particular, both functions are entire and according to [1, (9.1.16)] their Wronskian is given by

$$
W\left(\theta_{l}(z), \phi_{l}(z)\right)=1 \text {. }
$$

The eigenvalues of $H_{l}$ with a Dirichlet boundary condition at $x=1$ are given by the zeros of the entire function $\phi_{l}(z, 1)$ which are the squares of the positive zeros of the Bessel function $J_{l+1 / 2}$ of order $l+1 / 2$ :

$$
\mu_{l, n}=\left(j_{l+1 / 2, n}\right)^{2} .
$$

Similarly, the eigenvalues of $H_{l}$ with the boundary condition $f^{\prime}(1)+\beta f(1)=0$ are given by the zeros of the entire function $\phi_{l}^{\prime}(z, 1)+\beta \phi_{l}(z, 1)$ and the positive eigenvalues are the squares of the positive zeros of $z J_{l+3 / 2}(z)-(\beta+l+1) J_{l+1 / 2}(z)$ (cf. [17, §3.2]):

$$
\lambda_{l, n}^{\beta}=\left(j_{l+1 / 2, n}^{\beta}\right)^{2} .
$$

Here the eigenvalues are counted according to $\lambda_{l, 0}^{\beta}<\mu_{l, 1}<\lambda_{l, 1}^{\beta}<\ldots$ In particular, observe that the first eigenvalue $\lambda_{l, 0}^{\beta}$ will be zero for $\beta=-(l+1)$ and negative for $\beta<-(l+1)$.

Note that asymptotically $([1,(9.5 .12)])$

$$
\begin{aligned}
& \sqrt{\mu_{l, n}}=j_{l+\frac{1}{2}, n}=\left(n+\frac{l}{2}\right) \pi+O\left(n^{-1}\right), \\
& \sqrt{\lambda_{l, n}^{\beta}}=j_{l+\frac{1}{2}, n}^{\beta}=j_{l+\frac{3}{2}, n}+O\left(n^{-1}\right)=\left(n+\frac{l+1}{2}\right) \pi+O\left(n^{-1}\right)
\end{aligned}
$$

for fixed $l$.

Now let us look at perturbations

$$
H=H_{l}+q(x)
$$

assuming that the potential $q$ satisfies the following conditions:

Hypothesis 2.1. Let $l \in\left[-\frac{1}{2}, \infty\right)$. Set

$$
\tilde{q}(x)= \begin{cases}|q(x)|, & l>-\frac{1}{2} \\ (1-\log (x))|q(x)|, & l=-\frac{1}{2}\end{cases}
$$

and suppose $q$ is real-valued such that

$$
x \tilde{q}(x) \in L^{1}(0,1) .
$$


Lemma 2.2. Assume Hypothesis 2.1. Then there is a solution $\phi(z, x)$ of $H f=z f$ which is entire with respect to $z$ and satisfies the integral equation

$$
\phi(z, x)=\phi_{l}(z, x)+\int_{0}^{x} G_{l}(z, x, y) q(y) \phi(z, y) d y
$$

where

$$
G_{l}(z, x, y)=\phi_{l}(z, x) \theta_{l}(z, y)-\phi_{l}(z, y) \theta_{l}(z, x)
$$

is the Green function of the initial value problem. Moreover, this solution satisfies the estimate

$$
\left|\phi(z, x)-\phi_{l}(z, x)\right| \leq C\left(\frac{x}{1+|z|^{1 / 2} x}\right)^{l+1} \mathrm{e}^{\left|\operatorname{Im}\left(z^{1 / 2}\right)\right| x} \int_{0}^{x} \frac{y \tilde{q}(y)}{1+|z|^{1 / 2} y} d y .
$$

The derivative is given by

$$
\phi^{\prime}(z, x)=\phi_{l}^{\prime}(z, x)+\int_{0}^{x} \frac{\partial}{\partial x} G_{l}(z, x, y) q(y) \phi(z, y) d y
$$

and satisfies the estimate

$$
\left|\phi^{\prime}(z, x)-\phi_{l}^{\prime}(z, x)\right| \leq C\left(\frac{x}{1+|z|^{1 / 2} x}\right)^{l} \mathrm{e}^{\left|\operatorname{Im}\left(z^{1 / 2}\right)\right| x} \int_{0}^{x} \frac{y \tilde{q}(y)}{1+|z|^{1 / 2} y} d y .
$$

Proof. In a way similar to [6] (see also [14]) this can be shown by iteration using Lemmas A.1 and A.2. Namely, it is easy to show that $\phi$ given by

$$
\begin{aligned}
& \phi=\sum_{n=0}^{\infty} \phi_{l, n}, \quad \phi_{l, 0}:=\phi_{l}, \\
& \phi_{l, n+1}(z, x):=\int_{0}^{x} G_{l}(z, x, y) q(y) \phi_{l, n}(z, y) d y, \quad n \in \mathbb{N}_{0},
\end{aligned}
$$

satisfies (2.16). The inequalities

$$
\left|\phi_{l, n}(z, x)\right| \leq \frac{C^{n+1}}{n !}\left(\frac{x}{1+|z|^{1 / 2} x}\right)^{l+1} \mathrm{e}^{\left|\operatorname{Im}\left(z^{1 / 2}\right)\right| x}\left(\int_{0}^{x} \frac{y \tilde{q}(y)}{1+|z|^{1 / 2} y} d y\right)^{n},
$$

which are necessary to prove the convergence in (2.21), follow by induction from (2.15) and Lemma A.1. By (2.21) and (2.23), we get inequality (2.18), where $\tilde{q}$ is given in (2.14). Hear $C$ is to be understood as a generic constant whose value is different in (2.18) and in Lemma A.1. The properties of $\phi^{\prime}$ follow in a quite similar way from (2.21) - (2.23) and Lemma A.2. Finally, using (2.8), (2.16), (2.17), and (2.19) one can see that $H \phi=z \phi$.

Using the well-known asymptotic formulas for Bessel function [1, (9.2.1), (9.2.11)] we obtain

$$
\begin{aligned}
& \phi(z, x)=z^{-\frac{l+1}{2}}\left(\sin \left(\sqrt{z} x-\frac{l \pi}{2}\right)+O\left(|z|^{-1 / 2} \mathrm{e}^{x|\operatorname{Im}(\sqrt{z})|}\right)\right), \\
& \phi^{\prime}(z, x)=z^{-\frac{l}{2}}\left(\cos \left(\sqrt{z} x-\frac{l \pi}{2}\right)+O\left(|z|^{-1 / 2} \mathrm{e}^{x|\operatorname{Im}(\sqrt{z})|}\right)\right),
\end{aligned}
$$

as $z \rightarrow \infty$. Next, note that

$$
\lim _{x \rightarrow 0} x^{-l-1} \phi(z, x)=\lim _{x \rightarrow 0} x^{-l-1} \phi_{l}(z, x)=\frac{\sqrt{\pi}}{\Gamma\left(l+\frac{3}{2}\right) 2^{l+1}} .
$$


Moreover, using d'Alembert's formula (cf. [8, Sect. XI.6]) a second linearly independent solution, satisfying $W(\theta(z), \phi(z))=1$, is given by

$$
\theta(z, x)=-\phi(z, x) \int_{x}^{c} \frac{d y}{\phi(z, y)^{2}} .
$$

where $c=c(z)$ has to be chosen such that $\phi(z, x)$ does not vanish in $(0, c)$. In particular it is straightforward to show

Corollary 2.3. Assume Hypothesis [2.1. The differential equation $H f=z f$ has two linearly independent solutions, satisfying $W(\theta(z), \phi(z))=1$, of the form

$$
\phi(z, x)=x^{l+1} \tilde{\phi}(z, x), \quad \theta(z, x)= \begin{cases}\frac{x^{-l}}{2 l+1} \tilde{\theta}(z, x), & l>-\frac{1}{2}, \\ -x^{1 / 2} \log (x) \tilde{\theta}(z, x), & l=-\frac{1}{2},\end{cases}
$$

where $\tilde{\phi}(z, x), \tilde{\theta}(z, x) \in C(\mathbb{C},[0,1])$ are jointly continuous and $\tilde{\phi}(z, 0)=\tilde{\theta}(z, 0)^{-1} \neq$ 0 .

Unfortunately, since $c(z) \rightarrow 0$ as $z \rightarrow \infty$, this simple approach to get a second solution looses control over $\theta(z, x)$ as a function of $z$. In particular, it is not clear that this second solution can be chosen to be entire as a function of $z$. We will construct a second solution with better control with respect to $z$ in Lemma 2.6 below.

In any case, the behavior of the solutions of $H f=z f$ near $x=0$ implies

Theorem 2.4. Assume Hypothesis [2.1. The differential equation $H=H_{l}+q$ is limit circle at $x=0$ if $l \in\left[-\frac{1}{2}, \frac{1}{2}\right)$ and limit point at $x=0$ for $l \geq \frac{1}{2}$. In particular, $H$ associated with the boundary conditions at $x=0$ (for $l \in\left[-\frac{1}{2}, \frac{1}{2}\right)$ ) and $x=1$

$$
\lim _{x \rightarrow 0} x^{l}\left((l+1) f(x)-x f^{\prime}(x)\right)=0, \quad f(1)=0 \text { or } f^{\prime}(1)+\beta f(1)=0
$$

is self-adjoint. Moreover, the spectrum of $H$ is purely discrete and bounded from below.

Proof. Since $\phi(z, x)$ and $\theta(z, x)$ are both in $L^{2}(0,1)$ if and only if $l \in\left[-\frac{1}{2}, \frac{1}{2}\right)$ we deduce that $H$ is limit circle at $x$ if and only if $l \in\left[-\frac{1}{2}, \frac{1}{2}\right)$. Moreover, in this case we can choose the boundary condition (cf. [15, Sect 9.2])

$$
\lim _{x \rightarrow 0} W(\phi(0, x), f(x))=\frac{\sqrt{\pi}}{\Gamma\left(l+\frac{3}{2}\right) 2^{l+1}} \lim _{x \rightarrow 0} x^{l}\left((l+1) f(x)-x f^{\prime}(x)\right)=0,
$$

where we have used (2.26) and the fact that any solution in the maximal domain of the differential expression satisfies $\lim _{x \rightarrow 0} x^{l+1} f(x)=0$.

Furthermore, since $\phi(z, x)$ has only a finite number of zeros inside $(0,1)$, the differential expression is nonoscillatory for every $z \in \mathbb{R}$, we conclude that the spectrum is purely discrete and bounded from below (cf. [16, Thm. 14.9]).

Now we are able to prove our eigenvalue asymptotics using a refined version of the approach by Pöschel and Trubowitz [1].

Theorem 2.5. Assume Hypothesis 2.1, Then the Dirichlet eigenvalues corresponding to the boundary condition $f(1)=0$ satisfy

$$
\mu_{n}=\left(j_{l+\frac{1}{2}, n}+\varepsilon_{n}\right)^{2}
$$


where

$$
\varepsilon_{n}=O\left(\int_{0}^{1} \frac{y \tilde{q}(y)}{1+n y} d y\right) .
$$

Similarly the eigenvalues corresponding to the boundary condition $f^{\prime}(1)+\beta f(1)=0$ satisfy

$$
\lambda_{n}^{\beta}=\left(j_{l+\frac{1}{2}, n}^{\beta}+\tilde{\varepsilon}_{n}\right)^{2}
$$

where $\tilde{\varepsilon}_{n}$ is of the same order as $\varepsilon_{n}$.

Proof. We set $\phi_{l}(z):=\phi_{l}(z, 1)=\sqrt{\pi / 2} z^{-\frac{2 l+1}{4}} J_{l+1 / 2}(\sqrt{z})$ and $\phi(z)=\phi(z, 1)$. Then our estimate (2.18) reads

$$
\left|\phi(z)-\phi_{l}(z)\right| \leq C \frac{\mathrm{e}^{|\operatorname{Im} \sqrt{z}|}}{(1+\sqrt{|z|})^{l+1}} \varepsilon(z), \quad \varepsilon(z):=\int_{0}^{1} \frac{y \tilde{q}(y)}{1+|z|^{1 / 2} y} d y .
$$

Next, using [1, (9.2.1), (9.2.11)], we have

$$
\begin{aligned}
& \phi_{l}(z)=z^{-\frac{l+1}{2}}\left(\sin \left(\sqrt{z}-\frac{l \pi}{2}\right)+O\left(|z|^{-1 / 2} \mathrm{e}^{\left|\operatorname{Im}\left(z^{1 / 2}\right)\right|}\right)\right), \\
& \dot{\phi}_{l}(z)=\frac{1}{2} z^{-\frac{l+2}{2}}\left(\cos \left(\sqrt{z}-\frac{l \pi}{2}\right)+O\left(|z|^{-1 / 2} \mathrm{e}^{\left|\operatorname{Im}\left(z^{1 / 2}\right)\right|}\right)\right),
\end{aligned}
$$

where the dot denotes a derivative with respect to $z$. Hence, taking into account (2.11), (2.32), and (2.33), we get

$$
\phi_{l}\left(\left(j_{l+\frac{1}{2}, n}\right)^{2}\right)=0, \quad \dot{\phi}_{l}\left(\left(j_{l+\frac{1}{2}, n}\right)^{2}\right)=\frac{1}{2}\left(j_{l+\frac{1}{2}, n}\right)^{-(l+2)}\left((-1)^{n}+O\left(n^{-1}\right)\right) .
$$

Furthermore, (2.32)-(2.34) together with the mean value theorem yield (for sufficiently large $n$ )

$$
\begin{gathered}
\left|\phi_{l}\left(\left(j_{l+\frac{1}{2}, n} \pm \varepsilon_{n}\right)^{2}\right)\right| \geq \frac{1}{4}\left(j_{l+\frac{1}{2}, n}+\varepsilon_{n}\right)^{-(l+2)}\left|\left(j_{l+\frac{1}{2}, n} \pm \varepsilon_{n}\right)^{2}-j_{l+\frac{1}{2}, n}^{2}\right| \\
\geq \frac{1}{4} \frac{\varepsilon_{n}\left|2 j_{l+\frac{1}{2}, n}-\varepsilon_{n}\right|}{\left(j_{l+\frac{1}{2}, n}+\varepsilon_{n}\right)^{l+2}}>\frac{1}{4} \frac{\varepsilon_{n}}{\left(j_{l+\frac{1}{2}, n}+1\right)^{l+1}}=2 C \frac{\varepsilon\left(j_{l+\frac{1}{2}, n}\right)}{\left(j_{l+\frac{1}{2}, n}+1\right)^{l+1}},
\end{gathered}
$$

where $\varepsilon_{n}=8 C \varepsilon\left(j_{l+\frac{1}{2}, n}\right)$. Thus

$$
\begin{aligned}
\left|\phi\left(\left(j_{l+\frac{1}{2}, n} \pm \varepsilon_{n}\right)^{2}\right)-\phi_{l}\left(\left(j_{l+\frac{1}{2}, n} \pm \varepsilon_{n}\right)^{2}\right)\right| \leq & C \frac{\varepsilon\left(j_{l+\frac{1}{2}, n}-\varepsilon_{n}\right)}{\left(1+j_{l+\frac{1}{2}, n}-\varepsilon_{n}\right)^{l+1}} \\
& <\left|\phi_{l}\left(\left(j_{l+\frac{1}{2}, n} \pm \varepsilon_{n}\right)^{2}\right)\right| .
\end{aligned}
$$

This shows that $\phi(z)$ has different signs at $\left(j_{l+\frac{1}{2}, n}-\varepsilon_{n}\right)^{2}$ and $\left(j_{l+\frac{1}{2}, n}+\varepsilon_{n}\right)^{2}$, and thus there is at least one zero in between.

Let us show that $\phi$ has no other zeros. Since $|\sin z|>\frac{1}{4} \mathrm{e}^{|\operatorname{Im} z|}$ if $|z-\pi n| \geq \frac{\pi}{4}$ for all $n \in \mathbb{Z}$ (see for instance [11, Lemma 2.1]), there exists a $N \in \mathbb{N}$ such that

$$
\left|\phi_{l}(z)\right| \geq C_{l} \frac{\mathrm{e}^{|\operatorname{Im} \sqrt{z}|}}{|z|^{\frac{l+1}{2}}}, \quad|z| \geq N, \quad\left|\sqrt{z}-\pi n-\frac{l \pi}{2}\right| \geq \frac{\pi}{4}, \quad n \geq N,
$$

with some positive constant $C_{l}>0$ independent of $z$ (this estimate can also be deduced from [9, Lemma 22.1]). Since $\varepsilon(z) \rightarrow 0$ as $|z| \rightarrow \infty$, there exists $K>0$ 
such that $\varepsilon(z)<\left(C_{l} C\right)^{-1}$ if $|z|>K$. Thus, on contours $|z|=\left(K+N+\frac{l+1}{2}\right) \pi$ and $\left|\sqrt{z}-\pi n-\frac{l \pi}{2}\right|=\frac{\pi}{4}, n \geq K+N+1$, we obtain

$$
\left|\phi(z)-\phi_{l}(z)\right|<\left|\phi_{l}(z)\right| \text {. }
$$

By Rouché's theorem, $\phi(z)$ has as many roots as $\phi_{l}(z)$ in each of the bounded regions and the remaining unbounded region. Since all roots are simple, we are done.

An analogous argument can be given for the general eigenvalues based on zeros of

$$
\phi_{l}^{\beta}(z)=\phi_{l}^{\prime}(z, 1)+\beta \phi_{l}(z, 1)=\sqrt{\frac{\pi}{2}} z^{-\frac{2 l+1}{4}}\left((\beta+l+1) J_{l+\frac{1}{2}}(\sqrt{z})-\sqrt{z} J_{l+\frac{3}{2}}(\sqrt{z})\right) .
$$

Similar results for $q \in L^{2}$ were given by Guillot and Ralston [6], Carlson [4, Serier [14. Note that the case $x q(x) \in L^{p}$ for $l \in \mathbb{N}$ is covered in [2, 12, 13, for $l=0$ and in [3] for $l \in \mathbb{N}_{0}$, where the direct and inverse spectral problems have been effectively studied for operators with distributional potentials $q \in W^{-1, p}(0,1)$.

Finally we come to uniqueness results for the inverse problem. Considering the solution

$$
\psi_{l}^{\infty}(z, x)=\theta_{l}(z, 1) \phi_{l}(z, x)-\phi_{l}(z, 1) \theta_{l}(z, x)=G_{l}(z, x, 1)
$$

satisfying the initial conditions $\left(\psi_{l}^{\infty}(z, 1),\left(\psi_{l}^{\infty}(z, 1)\right)^{\prime}\right)=(0,1)$ and

$$
\begin{aligned}
\psi_{l}^{\beta}(z, x) & =-\left(\beta \theta_{l}(z, 1)+\theta_{l}^{\prime}(z, 1)\right) \phi_{l}(z, x)+\left(\beta \phi_{l}(z, 1)+\phi_{l}^{\prime}(z, 1)\right) \theta_{l}(z, x) \\
& =-\beta G_{l}(z, x, 1)+\frac{\partial G_{l}}{\partial y}(z, x, 1)
\end{aligned}
$$

satisfying the initial conditions $\left(\psi_{l}^{\beta}(z, 1),\left(\psi_{l}^{\beta}(z, 1)\right)^{\prime}\right)=(1,-\beta)$, we obtain the ana$\log$ of Lemma 2.2

Lemma 2.6. Assume Hypothesis 2.1. Then there is a solution $\psi^{\beta}(z, x)$ of $H u=z u$ which is entire with respect to $z$ and satisfies the integral equation

$$
\psi^{\beta}(z, x)=\psi_{l}^{\beta}(z, x)-\int_{x}^{1} G_{l}(z, x, y) q(y) \psi^{\beta}(z, y) d y .
$$

Moreover, for $l>-1 / 2$ this solution satisfies the estimate

$$
\begin{aligned}
& \left|\psi^{\infty}(z, x)-\psi_{l}^{\infty}(z, x)\right| \leq C\left(\frac{1+|z|^{1 / 2} x}{x+|z|^{1 / 2} x}\right)^{l} \frac{\mathrm{e}^{\left|\operatorname{Im}\left(z^{1 / 2}\right)\right|(1-x)}}{1+|z|^{1 / 2}} \int_{x}^{1} \frac{y \tilde{q}(y)}{1+|z|^{1 / 2} y} d y \\
& \left|\psi^{\beta}(z, x)-\psi_{l}^{\beta}(z, x)\right| \leq C\left(\frac{1+|z|^{1 / 2} x}{x+|z|^{1 / 2} x}\right)^{l} \mathrm{e}^{\left|\operatorname{Im}\left(z^{1 / 2}\right)\right|(1-x)} \int_{x}^{1} \frac{y \tilde{q}(y)}{1+|z|^{1 / 2} y} d y .
\end{aligned}
$$

In the case $l=-\frac{1}{2}$ an additional factor $(1-\log (x))$ appears in the right-hand sides of (2.38) and (2.39). 
Proof. Suppose $\beta \neq \infty$ first. In a way quite similar to the proof of Lemma 2.2 one can show that the solution $\psi^{\beta}$ admits representation

$$
\begin{aligned}
& \psi^{\beta}=\sum_{n=0}^{\infty} \psi_{l, n}^{\beta}, \quad \psi_{l, 0}^{\beta}:=\psi_{l}^{\beta}, \\
& \psi_{l, n+1}^{\beta}(z, x):=-\int_{x}^{1} G_{l}(z, x, y) q(y) \psi_{l, n}^{\beta}(z, y) d y \quad\left(n \in \mathbb{N}_{0}\right),
\end{aligned}
$$

where the functions $\psi_{l, n}^{\beta}$ satisfy inequalities

$$
\left|\psi_{l, n}^{\beta}(z, x)\right| \leq \frac{C^{n+1}}{n !}\left(\frac{1+|z|^{1 / 2} x}{x+|z|^{1 / 2} x}\right)^{l} \mathrm{e}^{\left|\operatorname{Im}\left(z^{1 / 2}\right)\right|(1-x)}\left(\int_{x}^{1} \frac{y \tilde{q}(y)}{1+|z|^{1 / 2} y} d y\right)^{n}
$$

for $l>-\frac{1}{2}$ and inequalities

$$
\begin{aligned}
\left|\psi_{l, n}^{\beta}(z, x)\right| & \leq \frac{C^{n+1}}{n !}(1-\log (x))\left(\frac{x+|z|^{1 / 2} x}{1+|z|^{1 / 2} x}\right)^{1 / 2} \\
& \times \mathrm{e}^{\left|\operatorname{Im}\left(z^{1 / 2}\right)\right|(1-x)}\left(\int_{x}^{1} \frac{y \tilde{q}(y)}{1+|z|^{1 / 2} y} d y\right)^{n}
\end{aligned}
$$

for $l=-\frac{1}{2}$. Indeed, the inequality (2.42) for $n=0$ and for some $C>0$ follows from (2.36), (A.22), and the fact that according to (2.17) we have $G_{l}(z, x, y)=$ $-G_{l}(z, y, x)$. Next, we use (2.41) and (A.2) to prove (2.42) for all $n$ by induction. By (2.40) - 2.42) we get (2.37) and (2.39). Finally, according to (2.37) we have $H \psi^{\beta}=z \psi^{\beta}$ and $\left(\psi^{\beta}(z, 1),\left(\psi^{\beta}(z, 1)\right)^{\prime}\right)=\left(\psi_{l}^{\beta}(z, 1),\left(\psi_{l}^{\beta}(z, 1)\right)^{\prime}\right)=(1,-\beta)$.

The inequality (2.43) for $n=0$ follows from (2.36) and (A.23), and the remaining part of the proof for $l=-1 / 2$ is analogous to the case $l>-1 / 2$.

The case $\beta=\infty$ can be treated in a similar way, it suffices to note that

$$
\left|\psi_{l, 0}^{\infty}(z, x)\right| \leq \frac{C}{1+|z|^{1 / 2}}\left(\frac{1+|z|^{1 / 2} x}{x+|z|^{1 / 2} x}\right)^{l} \mathrm{e}^{\left|\operatorname{Im}\left(z^{1 / 2}\right)\right|(1-x)}
$$

for $l>-\frac{1}{2}$ and

$$
\left|\psi_{-1 / 2,0}^{\infty}(z, x)\right| \leq(1-\log (x)) \frac{C}{1+|z|^{1 / 2}}\left(\frac{x+|z|^{1 / 2} x}{1+|z|^{1 / 2} x}\right)^{1 / 2} \mathrm{e}^{\left|\operatorname{Im}\left(z^{1 / 2}\right)\right|(1-x)}
$$

for $l=\frac{1}{2}$.

Remark 2.7. In [18] a second solution $\tilde{\psi}(z, x)$ is constructed by considering

$$
\tilde{\psi}(z, x)=\theta_{l}(z, x)-\int_{x}^{1} G_{l}(z, x, y) q(y) \tilde{\psi}(z, y) d y .
$$

It is claimed to be linearly independent for all $z$ and a reference is made to [6], where the corresponding claim for $l=1$ was made. However, this is wrong as the following counter example shows (a similar counter example was communicated to us by Ralston; see also []):

The function $\tilde{\psi}$ satisfies $\left(\tilde{\psi}(z, 1), \tilde{\psi}^{\prime}(z, 1)\right)=\left(\theta_{l}(z, 1), \theta_{l}^{\prime}(z, 1)\right)$. Let $y_{l+1 / 2, n}$ be a zero of $\theta_{l}(1, z)$ and chose $q(x)$ such that $y_{l+1 / 2, n}$ is an Dirichlet eigenvalue of $H$. Then $W\left(\tilde{\psi}\left(y_{l+1 / 2, n}\right), \phi\left(y_{l+1 / 2, n}\right)\right)=0$.

Now we come to our uniqueness results: 
Theorem 2.8. Assume Hypothesis 2.1. The following set of spectral data determine $q$ and $l$ uniquely:

(i) Two sets of eigenvalues $\lambda_{n}^{\alpha}$ and $\lambda_{n}^{\beta}$ for $\alpha \neq \beta$ (including the case $\lambda_{n}^{\infty}=\mu_{n}$ ).

(ii) The Dirichlet eigenvalues $\left\{\mu_{n}\right\}_{n=1}^{\infty}$ together with the norming constants

$$
\gamma_{n}^{-1}=\frac{1}{\phi^{\prime}\left(\mu_{n}, 1\right)^{2}} \int_{0}^{1} \phi\left(\mu_{n}, x\right)^{2} d x=-\frac{\dot{\phi}\left(\mu_{n}, 1\right)}{\phi^{\prime}\left(\mu_{n}, 1\right)}
$$

or the set of eigenvalues $\left\{\lambda_{n}^{\beta}\right\}_{n=0}^{\infty}$ together with the norming constants

$$
\left(\gamma_{n}^{\beta}\right)^{-1}=\frac{1}{\phi^{\beta}\left(\lambda_{n}^{\beta}\right)^{2}} \int_{0}^{1} \phi\left(\lambda_{n}^{\beta}, x\right)^{2} d x=\frac{\dot{\phi^{\beta}}\left(\lambda_{n}^{\beta}\right)}{\left(1+\beta^{2}\right) \phi\left(\lambda_{n}^{\beta}, 1\right)},
$$

where $\phi^{\beta}(z):=\phi^{\prime}(z, 1)+\beta \phi(z, 1)$.

(iii) The eigenvalues $\lambda_{n}^{\beta}$ together with $\phi\left(\lambda_{n}^{\beta}, 1\right)$ if $\beta \neq \infty$ or $\phi^{\prime}\left(\lambda_{n}^{\beta}, 1\right)$ if $\beta \neq 0$.

Here the dot and prime denote derivatives with respect to $z$ and $x$, respectively.

Proof. (i) To see the first claim recall the Weyl $m$-function of $H$ at the regular endpoint $x=1$, which is given by

$$
m_{\beta}(z)=\frac{\phi(z, 1)-\beta \phi^{\prime}(z, 1)}{\phi^{\prime}(z, 1)+\beta \phi(z, 1)}
$$

and consider

$$
\tilde{m}(z)=m_{\beta}(z)-\frac{\alpha \beta+1}{\beta-\alpha}=\frac{1+\beta^{2}}{\alpha-\beta} \cdot \frac{\phi(z, 1)+\alpha \phi^{\prime}(z, 1)}{\phi^{\prime}(z, 1)+\beta \phi(z, 1)} .
$$

Then the well-known asymptotics [15]

$$
m_{\beta}(z)= \begin{cases}-\sqrt{-z}+O(1), & \beta=\infty, \\ \beta+o(1), & \beta \in \mathbb{R},\end{cases}
$$

show that $\tilde{m}(z)$ is uniquely determined by its zeros and poles via its Hadamard product. To complete the proof of (i) it suffices to note that the Weyl function $m_{\beta}(z)$ uniquely determines the potential $q$ and the constants $l$ and $\beta$ (see [10]).

(ii) Similarly, the Herglotz function $m_{\beta}(z)$ is uniquely determined by its poles $\lambda_{n}^{\beta}$ and residues $-\gamma_{n}^{\beta}$.

(iii) Let $\beta \neq \infty$ and $\phi\left(\lambda_{n}^{\beta}, 1\right)$ be given. Clearly $l$ can be read off by the asymptotics of $\lambda_{n}^{\beta}$. So let us consider $H$ and $\hat{H}$ associated with $q$ and $\hat{q}$, but with the same $l$. Now, following [11, Chapter 3] consider the meromorphic function

$$
\frac{(\phi(z, x)-\hat{\phi}(z, x))\left(\psi^{\beta}(z, x)-\hat{\psi}^{\beta}(z, x)\right)}{\phi^{\beta}(z)} .
$$

The only poles are at the eigenvalues $\lambda_{n}^{\beta}$ and the residues are given by

$$
\frac{\left(\phi\left(\lambda_{n}^{\beta}, x\right)-\hat{\phi}\left(\lambda_{n}^{\beta}, x\right)\right)^{2}}{\phi\left(\lambda_{n}^{\beta}, 1\right) \dot{\phi}^{\beta}\left(\lambda_{n}^{\beta}\right)} \geq 0,
$$


where we have used $\phi\left(\lambda_{n}^{\beta}, x\right)=\phi\left(\lambda_{n}^{\beta}, 1\right) \psi^{\beta}\left(\lambda_{n}^{\beta}, x\right)$ together with our assumption $\phi\left(\lambda_{n}^{\beta}, 1\right)=\hat{\phi}\left(\lambda_{n}^{\beta}, 1\right)$. Using our estimates (2.18), (2.20), and (2.39), we have

$$
\begin{gathered}
|\phi(z, x)-\hat{\phi}(z, x)| \leq C\left(\frac{x}{1+|z|^{1 / 2} x}\right)^{l+1} \mathrm{e}^{\left|\operatorname{Im}\left(z^{1 / 2}\right)\right| x} \int_{0}^{x} \frac{y(\tilde{q}(y)+\tilde{\hat{q}}(y))}{1+|z|^{1 / 2} y} d y, \\
\left|\psi^{\beta}(z, x)-\hat{\psi}^{\beta}(z, x)\right| \leq C\left(\frac{1+|z|^{1 / 2} x}{x+|z|^{1 / 2} x}\right)^{l} \mathrm{e}^{\left|\operatorname{Im}\left(z^{1 / 2}\right)\right|(1-x)} \int_{x}^{1} \frac{y(\tilde{q}(y)+\tilde{\hat{q}}(y))}{1+|z|^{1 / 2} y} d y .
\end{gathered}
$$

Further, together with the estimate from the proof of Theorem 2.5. we get

$$
\left|\phi^{\beta}(z)\right| \geq\left|\phi_{l}^{\beta}(z)\right|-\left|\phi^{\beta}(z)-\phi_{l}^{\beta}(z)\right|>\frac{1}{2}\left|\phi_{l}^{\beta}(z)\right| \geq C_{l} \frac{\mathrm{e}^{\left|\operatorname{Im}(z)^{1 / 2}\right|}}{|z|^{\frac{l}{2}}}
$$

for $|z|=r_{n}=(n+(l+1) / 2)^{2} \pi^{2}$ with $n$ sufficiently large. Observing that for every $x \in(0,1)$

$$
\int_{x}^{1} \frac{y(\tilde{q}(y)+\tilde{\hat{q}}(y))}{1+|z|^{1 / 2} y} d y \leq \frac{1}{1+|z|^{1 / 2} x} \int_{x}^{1} y(\tilde{q}(y)+\tilde{\hat{q}}(y)) d y=O\left(|z|^{-1 / 2}\right)
$$

we see that the function (2.47) is $o\left(r_{n}^{-1}\right)$ along these circles and thus Lemma 3.2 from [11] shows that the residues are all zero. Hence $\phi\left(\lambda_{1}^{\beta}, x\right)=\hat{\phi}\left(\lambda_{1}^{\beta}, x\right)$ implying $q=\hat{q}$.

If $\beta \neq 0$ and the set $\phi^{\prime}\left(\lambda_{n}^{\beta}, 1\right)$ is given, then the proof remains essentially the same. The only difference is that we now use $\phi\left(\lambda_{n}^{\beta}, x\right)=-\beta^{-1} \phi^{\prime}\left(\lambda_{n}^{\beta}, 1\right) \psi^{\beta}\left(\lambda_{n}^{\beta}, x\right)$ if $\beta \neq \infty$. In the case $\beta=\infty$ we need to use $\phi^{\beta}(z)=\phi^{\prime}(z, 1), \phi\left(\lambda_{n}^{\beta}, x\right)=\phi^{\prime}\left(\lambda_{n}^{\beta}, 1\right) \psi^{\beta}\left(\lambda_{n}^{\beta}, x\right)$, and the estimate (2.38) instead of (2.39).

\section{Appendix A. Some estimates for the Spherical Schrödinger Equation}

In this appendix we want to provide some estimates for the solutions of the spherical Schrödinger equation which are crucial for the main body of our paper. These results are due to Guillot and Ralston [6] in the case $l=1$. The analog estimates for arbitrary $l \geq-\frac{1}{2}$ have been stated in 18 without proof. However, the estimate (39) from [18] is clearly wrong in the case $l=-\frac{1}{2}$. The case of integer $l$ is given in [14. Since the proof in [14] (and [6]) uses the explicit representation of spherical Bessel functions in terms of trigonometric functions we have decided to provide the details for the general case in this appendix. We assume that $x \in(0,1)$.

Lemma A.1. For $l>-\frac{1}{2}$ the following estimates hold:

$$
\begin{aligned}
\left|\phi_{l}(z, x)\right| & \leq C\left(\frac{x}{1+|z|^{1 / 2} x}\right)^{l+1} \mathrm{e}^{\left|\operatorname{Im}\left(z^{1 / 2}\right)\right| x}, \\
\left|G_{l}(z, x, y)\right| & \leq C\left(\frac{x}{1+|z|^{1 / 2} x}\right)^{l+1}\left(\frac{1+|z|^{1 / 2} y}{y}\right)^{l} \mathrm{e}^{\left|\operatorname{Im}\left(z^{1 / 2}\right)\right|(x-y)}, \quad y \leq x .
\end{aligned}
$$

For the case $l=-\frac{1}{2}$ formula (A.1) remains valid and one has to replace (A.2) by

$$
\begin{aligned}
\left|G_{-1 / 2}(z, x, y)\right| \leq & C\left(\frac{x y}{\left(1+|z|^{1 / 2} x\right)\left(1+|z|^{1 / 2} y\right)}\right)^{1 / 2} \\
& \times \mathrm{e}^{\left|\operatorname{Im}\left(z^{1 / 2}\right)\right|(x-y)}(1-\log (y)), \quad y \leq x .
\end{aligned}
$$


Proof. First of all recall (2.4), (2.5), and (2.17), and note that for all $l \geq-\frac{1}{2}$

$$
\begin{aligned}
\phi_{l}(z, x) & =\sqrt{\frac{\pi x}{2}} z^{-\frac{2 l+1}{4}} J_{l+\frac{1}{2}}(\sqrt{z} x) \\
G_{l}(z, x, y) & =-\frac{\pi}{2} \sqrt{x y}\left(J_{l+\frac{1}{2}}(\sqrt{z} x) Y_{l+\frac{1}{2}}(\sqrt{z} y)-J_{l+\frac{1}{2}}(\sqrt{z} y) Y_{l+\frac{1}{2}}(\sqrt{z} x)\right) \\
& =-\frac{\pi \mathrm{i}}{4} \sqrt{x y}\left(H_{l+\frac{1}{2}}^{(1)}(\sqrt{z} x) H_{l+\frac{1}{2}}^{(2)}(\sqrt{z} y)-H_{l+\frac{1}{2}}^{(1)}(\sqrt{z} y) H_{l+\frac{1}{2}}^{(2)}(\sqrt{z} x)\right) .
\end{aligned}
$$

Here $H_{l+1 / 2}^{(1)}(z)=J_{l+1 / 2}(z)+\mathrm{i} Y_{l+1 / 2}(z)$ and $H_{l+1 / 2}^{(2)}(z)=J_{l+1 / 2}(z)-\mathrm{i} Y_{l+1 / 2}(z)$ are the Hankel functions of the first and second kind, respectively. Moreover, recall $Y_{l+1 / 2}(z)=\sin ((l+1 / 2) \pi)^{-1}\left(\cos ((l+1 / 2) \pi) J_{l+1 / 2}(z)-J_{-l-1 / 2}(z)\right)([1,(9.1 .2)])$.

The claimed estimates (A.1) and (A.2) can be shown by combining the following asymptotic expansions which yield estimates for $|z| \geq 1$ and $|z| \leq 1$, respectively. In fact, we have [1, (9.2.7)-(9.2.10)]

$$
\begin{array}{ll}
H_{l+\frac{1}{2}}^{(1)}(z)=\sqrt{\frac{2}{\pi z}} \mathrm{e}^{\mathrm{i}(z-(l+1) \pi / 2)}(1+O(1 / z)), & -\pi<\arg (z)<2 \pi, \\
H_{l+\frac{1}{2}}^{(2)}(z)=\sqrt{\frac{2}{\pi z}} \mathrm{e}^{-\mathrm{i}(z-(l+1) \pi / 2)}(1+O(1 / z)), & -2 \pi<\arg (z)<\pi,
\end{array}
$$

for $z \rightarrow \infty$ with the error uniform strictly inside the indicated sectors ([17, (7.2.1)(7.2.2)]). Using the power series for the Bessel and Neumann functions [1, (9.1.10)(9.1.11)] we get

$$
\begin{aligned}
& J_{l+\frac{1}{2}}(z)=\frac{1}{\Gamma\left(l+\frac{3}{2}\right)}\left(\frac{z}{2}\right)^{l+\frac{1}{2}}(1+O(z)), \\
& Y_{l+\frac{1}{2}}(z)= \begin{cases}-\frac{\Gamma\left(l+\frac{1}{2}\right)}{\pi}\left(\frac{z}{2}\right)^{-l-\frac{1}{2}}\left(1+O\left(z^{\min (1,2 l+1)}\right)\right), & l+\frac{1}{2}>0, \\
\frac{2}{\pi} \log (z)+O(1), & l=-\frac{1}{2},\end{cases}
\end{aligned}
$$

for $z \rightarrow 0$. Thus, (A.1) immediately follows from (A.6), (A.7), and (A.8). The estimate (A.2) can be also deduced from A.6 A.9. For instance, using (A.8), (A.9), and the fact that the function $x \mapsto x(1+x)^{-1}$ is increasing, we get for $|\eta| \leq|\xi| \leq 1$

$$
\begin{aligned}
& \left|J_{l+\frac{1}{2}}(\xi) Y_{l+\frac{1}{2}}(\eta)-J_{l+\frac{1}{2}}(\eta) Y_{l+\frac{1}{2}}(\xi)\right| \\
& \leq C\left(\left(\frac{|\xi|}{1+|\xi|}\right)^{l+\frac{1}{2}}\left(\frac{|\eta|}{1+|\eta|}\right)^{-l-\frac{1}{2}}+\left(\frac{|\eta|}{1+|\eta|}\right)^{l+\frac{1}{2}}\left(\frac{|\xi|}{1+|\xi|}\right)^{-l-\frac{1}{2}}\right) \\
& \quad \leq 2 C\left(\frac{|\xi|}{1+|\xi|}\right)^{l+\frac{1}{2}}\left(\frac{|\eta|}{1+|\eta|}\right)^{-l-\frac{1}{2}} .
\end{aligned}
$$

Similarly one handles the cases $|\eta| \leq 1 \leq|\xi|$ and $1 \leq|\eta| \leq|\xi|$ to obtain the desired result (for the last one use the expression in terms of Hankel functions).

The estimate (A.3) requires some further considerations, and we also split this case into three subcases: a) sufficiently large values of $|z|^{1 / 2} y$; b) the values of $|z|^{1 / 2} x$ are sufficiently large and the values of $|z|^{1 / 2} y$ are bounded; c) $|z|^{1 / 2} x$ is bounded. In all the subcases it is assumed that $y \leq x$. 
To prove (A.3) for the subcase of sufficiently large values of $|z|^{1 / 2} y$ one can use again the equality (A.5):

$$
G_{-1 / 2}(z, x, y)=-\frac{\pi \mathrm{i}}{4} \sqrt{x y}\left(H_{0}^{(1)}(\sqrt{z} x) H_{0}^{(2)}(\sqrt{z} y)-H_{0}^{(1)}(\sqrt{z} y) H_{0}^{(2)}(\sqrt{z} x)\right)
$$

Taking into account (A.6) and (A.7) we easily derive (A.3) in the subcase a).

Assume now that the values of $|z|^{1 / 2} y$ are bounded. Note that (see (2.5), (2.17)

$$
G_{-1 / 2}(z, x, y)=\phi_{-\frac{1}{2}}(z, x) \theta_{-\frac{1}{2}}(z, y)-\phi_{-\frac{1}{2}}(z, y) \theta_{-\frac{1}{2}}(z, x),
$$

where

$$
\theta_{-\frac{1}{2}}(z, x)=\sqrt{\frac{\pi x}{2}}\left(Y_{0}(\sqrt{z} x)-\frac{1}{\pi} \log (z) J_{0}(\sqrt{z} x)\right) .
$$

Using the integral representations [1, (9.1.18) and (9.1.19)], we have

$$
\theta_{-\frac{1}{2}}(z, x)=\pi^{-3 / 2} \sqrt{2 x} \int_{0}^{\pi} \cos (\sqrt{z} x \cos \theta)\left\{\gamma+\log (x)+\log \left(2 \sin ^{2} \theta\right)\right\} d \theta .
$$

It is immediate from (A.11) that

$$
\left|\theta_{-\frac{1}{2}}(z, x)\right| \leq C_{1} \sqrt{x}(1-\log (x)) \mathrm{e}^{\left|\operatorname{Im}\left(z^{1 / 2}\right)\right| x} .
$$

Further, since the values of $|z|^{1 / 2} y$ are bounded, by (A.1) and (A.12) we derive

$$
\begin{aligned}
& \left|\phi_{-\frac{1}{2}}(z, x) \theta_{-\frac{1}{2}}(z, y)\right| \leq C_{2}\left(\frac{x y}{1+|z|^{1 / 2} x}\right)^{1 / 2}(1-\log (y)) \mathrm{e}^{\left|\operatorname{Im}\left(z^{1 / 2}\right)\right| x} \\
& \leq C_{3}\left(\frac{x y}{\left(1+|z|^{1 / 2} x\right)\left(1+|z|^{1 / 2} y\right)}\right)^{1 / 2} \mathrm{e}^{\left|\operatorname{Im}\left(z^{1 / 2}\right)\right|(x-y)}(1-\log (y)) .
\end{aligned}
$$

For large values of $|z|^{1 / 2} x$, Hankel's asymptotic expansions [1, (9.2.5) and (9.2.6)] yield:

$$
\left|\theta_{-\frac{1}{2}}(z, x)\right| \leq C_{4}|z|^{-1 / 4}(1+\log (|z|)) \mathrm{e}^{\left|\operatorname{Im}\left(z^{1 / 2}\right)\right| x} .
$$

Since $|z|^{1 / 2} x$ is sufficiently large, the values $|z|$ and $\log (|z|)$ are also large. Moreover, $|z|^{1 / 2} y$ is bounded and hence $\log (|z|)+2 \log (y)$ is bounded from above. Thus, A.14 can be rewritten in the form

$$
\left|\theta_{-\frac{1}{2}}(z, x)\right| \leq C_{5}\left(\frac{x}{1+|z|^{1 / 2} x}\right)^{1 / 2} \mathrm{e}^{\left|\operatorname{Im}\left(z^{1 / 2}\right)\right| x}(1-\log (y)) .
$$

It follows from (A.1) and (A.15) that

$$
\begin{aligned}
\left|\theta_{-\frac{1}{2}}(z, x) \phi_{-\frac{1}{2}}(z, y)\right| \leq & C_{6}\left(\frac{x y}{\left(1+|z|^{1 / 2} x\right)\left(1+|z|^{1 / 2} y\right)}\right)^{1 / 2} \\
& \times \mathrm{e}^{\left|\operatorname{Im}\left(z^{1 / 2}\right)\right|(x-y)}(1-\log (y)) .
\end{aligned}
$$

Inequalities (A.13) and (A.16) yield (A.3) for the subcase b).

Finally, if $|z|^{1 / 2} x$ is bounded, according to (A.1) and (A.12) we have

$$
\left|\theta_{-\frac{1}{2}}(z, x) \phi_{-\frac{1}{2}}(z, y)\right| \leq C_{7} \sqrt{x y}(1-\log (x)) \leq C_{7} \sqrt{x y}(1-\log (y)) .
$$

Inequalities (A.13) and (A.17) imply (A.3) for the subcase c). 
To consider $\frac{\partial}{\partial x} \phi_{l}(z, x)$ and $\frac{\partial}{\partial x} G_{l}(z, x)$ one should simply use the asymptotic relations [1, (9.2.11)-(9.2.16)] on the derivatives of the special functions, in addition to the relations [1, (9.2.5)-(9.2.10)] which were used in the proof of the previous lemma. In particular, for sufficiently large values of $|z|$ we have

$$
\left|\frac{\partial}{\partial z} J_{l+\frac{1}{2}}(z)\right|+\left|\frac{\partial}{\partial z} Y_{l+\frac{1}{2}}(z)\right| \leq C_{8} \sqrt{\frac{2}{\pi z}} \mathrm{e}^{|\operatorname{Im}(z)|},
$$

and, as $z \rightarrow \infty$, we get

$$
\begin{aligned}
& \frac{\partial}{\partial z} H_{l+\frac{1}{2}}^{(1)}(z)=i \sqrt{\frac{2}{\pi z}} \mathrm{e}^{\mathrm{i}(z-(l+1) \pi / 2)}(1+O(1 / z)), \quad-\pi<\arg (z)<2 \pi \\
& \frac{\partial}{\partial z} H_{l+\frac{1}{2}}^{(2)}(z)=-i \sqrt{\frac{2}{\pi z}} \mathrm{e}^{-\mathrm{i}(z-(l+1) \pi / 2)}(1+O(1 / z)), \quad-2 \pi<\arg (z)<\pi .
\end{aligned}
$$

Lemma A.2. For $l>-\frac{1}{2}$ the following estimates hold:

$$
\left|\frac{\partial}{\partial x} \phi_{l}(z, x)\right| \leq C\left(\frac{x}{1+|z|^{1 / 2} x}\right)^{l} \mathrm{e}^{\left|\operatorname{Im}\left(z^{1 / 2}\right)\right| x},
$$

$$
\left|\frac{\partial}{\partial x} G_{l}(z, x, y)\right| \leq C\left(\frac{x}{1+|z|^{1 / 2} x}\right)^{l}\left(\frac{1+|z|^{1 / 2} y}{y}\right)^{l} \mathrm{e}^{\left|\operatorname{Im}\left(z^{1 / 2}\right)\right|(x-y)}, \quad y \leq x .
$$

For $l=-1 / 2$ formula (A.21) remains true and one has to replace (A.22) by

$$
\left|\frac{\partial}{\partial x} G_{-1 / 2}(z, x, y)\right| \leq C\left(\frac{\left.y+|z|^{1 / 2} x y\right)}{\left.x+|z|^{1 / 2} x y\right)}\right)^{1 / 2} \mathrm{e}^{\left|\operatorname{Im}\left(z^{1 / 2}\right)\right|(x-y)}(1-\log (y)), \quad y \leq x .
$$

Proof. The proof is similar to the proof of Lemma A.1 and we shall prove here only formula A.23). For the subcase of sufficiently large values of $|z|^{1 / 2} y$ we use the equality (A.10). Therefore, in view of (A.6), (A.7), (A.19), and (A.20) we derive

$$
\left|G_{-1 / 2}(z, x, y)\right| \leq C_{9} \mathrm{e}^{\left|\operatorname{Im}\left(z^{1 / 2}\right)\right|(x-y)}, \quad y \leq x,
$$

and A.23) is immediate.

When the values of $|z|^{1 / 2} y$ are bounded, formulas (A.12) and (A.21) imply the inequality

$$
\left|\frac{\partial}{\partial x} \phi_{-\frac{1}{2}}(z, x) \theta_{-\frac{1}{2}}(z, y)\right| \leq C_{10}\left(\frac{y+|z|^{1 / 2} x y}{x+|z|^{1 / 2} x y}\right)^{1 / 2} \mathrm{e}^{\left|\operatorname{Im}\left(z^{1 / 2}\right)\right|(x-y)}(1-\log (y)),
$$

which we shall use instead of A.13). For the subcase of sufficiently large values of $|z|^{1 / 2} x$ and bounded values of $|z|^{1 / 2} y$, it follows from (2.5), (A.15), and (A.18) that

$$
\left|\frac{\partial}{\partial x} \theta_{-\frac{1}{2}}(z, x)\right| \leq C_{11}\left(\frac{1+|z|^{1 / 2} x}{x}\right)^{1 / 2} \mathrm{e}^{\left|\operatorname{Im}\left(z^{1 / 2}\right)\right| x}(1-\log (y)) .
$$

Taking into account (A.1), (A.26), and boundedness of $|z|^{1 / 2} y$ we have

$$
\left|\frac{\partial}{\partial x} \theta_{-\frac{1}{2}}(z, x) \phi_{-\frac{1}{2}}(z, y)\right| \leq C_{12}\left(\frac{y+|z|^{1 / 2} x y}{\left.x+|z|^{1 / 2} x y\right)}\right)^{1 / 2} \mathrm{e}^{\left|\operatorname{Im}\left(z^{1 / 2}\right)\right|(x-y)}(1-\log (y)) .
$$


Inequalities A.25 and (A.27) yield (A.23) for the subcase of sufficiently large values of $|z|^{1 / 2} x$ and bounded values of $|z|^{1 / 2} y$.

Finally, if the values of $|z|^{1 / 2} x$ are bounded, formula (A.11) implies

$$
\left|\frac{\partial}{\partial x} \theta_{-\frac{1}{2}}(z, x)\right| \leq C_{13} x^{-1 / 2}(1-\log (x))
$$

and (A.27) follows from (A.1) and (A.28). By A.25) and (A.27) the inequality (A.23) holds again.

Acknowledgments. We thank Fritz Gesztesy and James Ralston for several helpful discussions. A.K. acknowledges the hospitality and financial support of the Erwin Schrödinger Institute and financial support from the IRCSET PostDoctoral Fellowship Program.

\section{REFERENCES}

[1] M. Abramovitz and I.A. Stegun, Handbook of Mathematical Functions, Dover, New York, 1972.

[2] S. Albeverio, R. Hryniv, and Ya. Mykytyuk, Inverse spectral problems for Sturm-Liouville operators in impedance form, J. Funct. Anal. 222, 143-177 (2005).

[3] S. Albeverio, R. Hryniv, and Ya. Mykytyuk, Inverse spectral problems for Bessel operators, J. Diff. Eqs. 241, 130-159 (2007).

[4] R. Carlson, Inverse spectral theory for some singular Sturm-Liouville problems, J. Diff. Eqs. 106, 121-140 (1993).

[5] R. Carlson, A Borg-Levinson theorem for Bessel operators, Pacific J. Math. 177, 1-26 (1997).

[6] J.-C. Guillot and J. V. Ralston, Inverse spectral theory for a singular Sturm-Liouville operator on [0,1], J. Diff. Eqs. 76, 353-373 (1988).

[7] J.-C. Guillot and J. V. Ralston, Erratum for: Inverse spectral theory for a singular SturmLiouville operator on [0,1], arXiv:1007.0713

[8] P. Hartman, Ordinary Differential Equations, 2nd ed., SIAM, Philadelphia, 2002.

[9] B. Ya. Levin, Lectures on Entire Functions, Transl. Math. Monographs 150, Amer. Math. Soc., Rhode Island, 1996.

[10] A. V. Marchenko, Sturm-Liouville Operators and Applications, Birkhäuser, Basel, 1986.

[11] J. Pöschel and E. Trubowitz, Inverse Spectral Theory, Pure and Applied Mathematics 130, Academic Press, Boston, 1987.

[12] A. M. Savchuk, A. A. Shkalikov, Sturm-Liouville operators with distribution potentials, Trans. Moscow Math. Soc. 2003, 143-192 (2003).

[13] A. M. Savchuk, A. A. Shkalikov, On the properties of maps connected with inverse SturmLiouville problems, Proc. Steklov. Inst. Math. 260, 218-237 (2008).

[14] F. Serier, The inverse spectral problem for radial Schrödinger operators on [0,1], J. Diff. Eqs. 235, 101-126 (2007).

[15] G. Teschl, Mathematical Methods in Quantum Mechanics; With Applications to Schrödinger Operators, Graduate Studies in Mathematics, Amer. Math. Soc., Rhode Island, 2009.

[16] J. Weidmann, Spectral Theory of Ordinary Differential Operators, Lecture Notes in Mathematics 1258, Springer, Berlin, 1987.

[17] G. N. Watson, A Treatise on the Theory of Bessel Functions, 2nd ed., Cambridge University Press, Cambridge, 1995.

[18] L. A. Zhornitskaya and V. S. Serov, Inverse eigenvalue problems for a singular SturmLiouville operator on [0,1], Inverse Problems 10:4, 975-987 (1994). 
Institute of Applied Mathematics and Mechanics, NAS of Ukraine, R. Luxemburg str. 74, Donetsk 83114, Ukraine, and School of Mathematical Sciences, Dublin Institute of Technology, Kevin Street, Dublin 8, Ireland

E-mail address: duzer80@gmail.com

Faculty of Mathematics, Nordbergstrasse 15, 1090 Wien, Austria

E-mail address: Oleksandr.Sakhnovych@univie.ac.at

$U R L:$ http://www.mat.univie.ac.at/ sakhnov/

Faculty of Mathematics, Nordbergstrasse 15, 1090 Wien, Austria, and International Erwin Schrödinger Institute for Mathematical Physics, Boltzmanngasse 9, 1090 Wien, Austria

E-mail address: Gerald.Teschl@univie.ac.at

$U R L:$ http://www.mat.univie.ac.at/ gerald/ 